\title{
Stability of Traffic Flow Networks with a Polytree Topology
}

\author{
Samuel Coogan ${ }^{\text {a }}$ Murat Arcak b \\ ${ }^{a}$ Department of Electrical Engineering, University of California, Los Angeles \\ ${ }^{\mathrm{b}}$ Department of Electrical Engineering and Computer Sciences, University of California, Berkeley
}

\begin{abstract}
We consider global stability of a flow network model for vehicular traffic. Standard approaches which rely on monotonicity of flow networks for stability analysis do not immediately apply to traffic networks with diverging junctions. In this paper, we show that the network model nonetheless exhibits a mixed monotonicity property. Mixed monotonicity allows us to prove global asymptotic stability by embedding the system in a larger system that is monotone.
\end{abstract}

\section{Introduction}

We study the dynamical behavior of a compartmental system model of traffic flow. The flow of mass from a compartment, or link, to downstream links is governed by a local flow demand as well as downstream supply of capacity available to accomodate incoming flow. Such an approach is well suited for modeling flow of vehicles on a freeway $[9,10,13,14]$.

This paper builds on recent results [5, 6, 13, 14, 23] which have utilized a compartmental systems approach [18] to analyze the dynamical behavior of transportation networks. In these prior works, the strongest results rely on the system dynamics being monotone whereby trajectories of the system preserve a partial ordering $[17,26]$. Yet, as detailed in Section 3.1, vehicular traffic networks with diverging junctions and fixed routing policies are not monotone, as noted in [6, 22], since downstream congestion on one outgoing link blocks incoming flow to neighboring outgoing links [24]. Thus, a significant gap exists in the literature for understanding the dynamics of traffic flow networks. The present paper works towards filling this gap.

We first show that traffic dynamics possess a mixed monotonicity property, which is much weaker than monotonicity. Despite the lack of monotonicity in the standard form of the dynamics, such systems can be embedded into a higher dimensional monotone system, $[11,15,20,25]$. With this key observation, we bring the

\footnotetext{
* e-mail: scoogan@ucla.edu, arcak@eecs.berkeley.edu. This work was supported by the NSF under grant CNS1446145.
}

powerful tools of monotone system theory to this more general problem.

Next, we identify a certain class of polytree networks for which, using this embedding, we prove global asymptotic convergence to a unique equilibrium. In exchange for the generality offered by mixed monotonicity, we obtain only a sufficient condition that requires equilibria of the embedding system to be unique. We show that this condition is satisfied for polytree networks. We show through an example that the polytree restriction is necessary for the embedding system to have a unique equilibrium.

In [7] and [8], we have noted that a similar discrete-time model of queue evolution in a network of signalized intersections is mixed monotone, but our focus was on finite state abstraction of the dynamics for automatic synthesis of control strategies rather than stability analysis. Furthermore, the model we consider for signalized intersections satisfies a sufficient condition for mixed monotonicity which is not generally satisfied by the freeway model considered here.

The remainder of the paper is organized as follows: In Section 2, we define the network model. In Section 3, we show that the dynamics are not monotone but are mixed monotone, which allows the system to be embedded in a larger dimensional monotone system. In Section 4, we apply these results to establish global stability for a class of networks, and we consider two examples. In Section 5, we provide a discussion of the approach and comparisons to other techniques for stability analysis of flow networks, and we give concluding remarks. 


\section{A Compartmental Model of Traffic Flow}

\subsection{Notation}

All inequalities are interpreted elementwise, e.g., for $x, y \in \mathbb{R}^{n}, x \leq y$ if and only if $x_{i} \leq y_{i}$ for $i=1, \ldots n$ where $x_{i}, y_{i}$ denote the $i$ th element of $x, y$. We denote the vector of all zeros by 0 when its dimension is clear from context. We denote the set of nonnegative real numbers by $\mathbb{R}_{\geq 0}=\{x \in \mathbb{R} \mid x \geq 0\}$.

\subsection{Network Topology}

We summarize the network model adopted here, which is based on the model we develop in [6]. A traffic network consists of a directed graph $\mathcal{G}=(\mathcal{V}, \mathcal{O})$ with junctions $\mathcal{V}$ and ordinary links $\mathcal{O}$ along with a set of entry links $\mathcal{R}$ which are entry points into the network. Let $\mathcal{L} \triangleq$ $\mathcal{O} \cup \mathcal{R}$. Physically, a link represents a segment of roadway, and we assume $\mathcal{G}$ is a connected graph. Let $\sigma(\ell)$ and $\tau(\ell)$ denote the head and tail junction of link $\ell \in \mathcal{L}$, respectively, where we assume $\sigma(\ell) \neq \tau(\ell)$, i.e., no selfloops. Traffic flows from $\tau(\ell)$ to $\sigma(\ell)$. By convention, $\tau(\ell)=\emptyset$ for all $\ell \in \mathcal{R}$.

For each $v \in \mathcal{V}$, we denote by $\mathcal{L}_{v}^{\text {in }} \subset \mathcal{L}$ the set of input links to node $v$ and by $\mathcal{L}_{v}^{\text {out }} \subset \mathcal{L}$ the set of output links, i.e. $\mathcal{L}_{v}^{\text {in }}=\{\ell: \sigma(\ell)=v\}$ and $\mathcal{L}_{v}^{\text {out }}=\{\ell: \tau(\ell)=v\}$. We assume $\mathcal{L}_{v}^{\text {in }} \neq \emptyset$ for all $v \in \mathcal{V}$, thus the network flow starts at entry links. Furthermore, we assume $\mathcal{L}_{\sigma(\ell)}^{\text {out }} \neq \emptyset$ for all $\ell \in \mathcal{R}$, i.e. entry links always flow into at least one ordinary link downstream. If $\left|\mathcal{L}_{v}^{\text {in }}\right|>1$, then $v$ is a merging junction, and if $\left|\mathcal{L}_{v}^{\text {out }}\right|>1$, then $v$ is a diverging junction.

Define $\mathcal{V}^{\text {sink }} \triangleq\left\{v \in \mathcal{V} \mid \mathcal{L}_{v}^{\text {out }}=\emptyset\right\}$ to be the set of junctions that have no outgoing links and

$$
\mathcal{L}^{\operatorname{sink}} \triangleq\left\{\ell \in \mathcal{L} \mid \sigma(\ell) \in \mathcal{V}^{\text {sink }}\right\}
$$

the corresponding set of input links to these junctions.

\subsection{Link supply and demand}

Each link $\ell \in \mathcal{L}$ has state $x_{\ell}(t) \in\left[0, \bar{x}_{\ell}\right]$ where $\bar{x}_{\ell} \in$ $(0, \infty)$ is the jam density of link $\ell$. Let $\bar{x}=\left\{\bar{x}_{\ell}\right\}_{\ell \in \mathcal{L}}$. Furthermore, each link possesses a state-dependent demand function $\Phi_{\ell}^{\text {out }}\left(x_{\ell}\right)$ and supply function $\Phi_{\ell}^{\text {in }}\left(x_{\ell}\right)$ satisfying:

Assumption 1 For each $\ell \in \mathcal{L}$ :

- The demand function $\Phi_{\ell}^{\text {out }}\left(x_{\ell}\right):\left[0, \bar{x}_{\ell}\right] \rightarrow \mathbb{R}_{\geq 0}$ is strictly increasing and Lipschitz continuous with $\Phi_{\ell}^{\text {out }}(0)=0$.

- The supply function $\Phi_{\ell}^{\mathrm{in}}\left(x_{\ell}\right):\left[0, \bar{x}_{\ell}\right] \rightarrow \mathbb{R}_{\geq 0}$ is strictly decreasing and Lipschitz continuous with $\Phi_{\ell}^{\mathrm{in}}\left(\bar{x}_{\ell}\right)=0$.

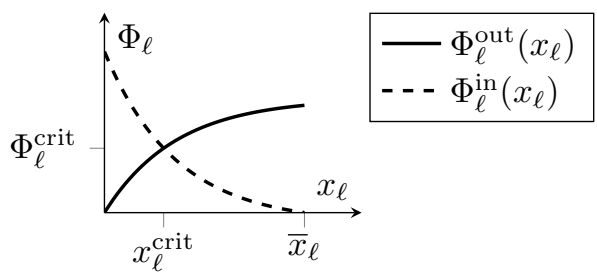

Fig. 1. Plot of prototypical supply and demand functions $\Phi_{\ell}^{\text {in }}\left(x_{\ell}\right)$ and $\Phi_{\ell}^{\text {out }}\left(x_{\ell}\right)$.

Assumption 1 implies that for each $\ell \in \mathcal{L}$, there exists unique $x_{\ell}^{\text {crit }}$ such that

$$
\Phi_{\ell}^{\text {out }}\left(x_{\ell}^{\text {crit }}\right)=\Phi_{\ell}^{\text {in }}\left(x_{\ell}^{\text {crit }}\right)=: \Phi_{\ell}^{\text {crit }}
$$

Figure 1 depicts examples of supply and demand functions satisfying Assumption 1. The demand of a link is interpreted as the maximum outflow of the link, and the supply of a link is interpreted as the maximum inflow of the link.

\subsection{Dynamic Model}

At each junction $v \in \mathcal{V}$, there exists a collection of fixed split ratios $\left\{\beta_{\rightarrow \ell}\right\}_{\ell \in \mathcal{L}_{v}^{\text {out }}}$ with each $\beta_{\rightarrow \ell}>0$ describing how incoming flow is split among outgoing links. Conservation of flow implies

$$
\sum_{\ell \in \mathcal{L}_{v}^{\text {out }}} \beta_{\rightarrow \ell} \leq 1 \quad \forall v \in \mathcal{V}
$$

where strict inequality in (3) implies that a fraction of the flow is routed off the network via, e.g., an unmodeled off-ramp.

Note that we associate a single split ratio with each output link rather than with each input/output link pair as in [6]. This implies that split ratios cannot differ for different incoming links and, as we will see in Section 3, leads to a mixed monotonicity property. If we assume each junction is either single-input or single-output as in $[10,23]$, then there is no distinction between the two models. 
The flow dynamics of the network are as follows:

$$
\begin{aligned}
& \dot{x}_{\ell}=f_{\ell}^{\text {in }}(x)-f_{\ell}^{\text {out }}(x) \quad \forall \ell \in \mathcal{L} \\
&=: F_{\ell}(x) \\
& \alpha^{v}(x) \triangleq \\
& \min \left\{1, \min _{\ell \in \mathcal{L}_{v}^{\text {out }}}\left\{\frac{\Phi_{\ell}^{\text {in }}\left(x_{\ell}\right)}{\beta_{\rightarrow \ell} \sum_{k \in \mathcal{L}_{v}^{\text {in }}} \Phi_{k}^{\text {out }}\left(x_{k}\right)}\right\}\right\} \forall v \in \mathcal{V} \\
& f_{\ell}^{\text {out }}(x)=\alpha^{\sigma(\ell)}(x) \Phi_{\ell}^{\text {out }}\left(x_{\ell}\right) \\
& f_{\ell}^{\text {in }}(x)= \begin{cases}\min \left\{d_{\ell}, \Phi_{\ell}^{\text {in }}\left(x_{\ell}\right)\right\} & \text { if } \ell \in \mathcal{R} \\
\beta_{\rightarrow \ell} \sum_{k \in \mathcal{L}_{\tau(\ell)}^{\text {in }}} f_{k}^{\text {out }}\left(x_{k}\right) & \text { if } \ell \in \mathcal{O} .\end{cases}
\end{aligned}
$$

Above, $\alpha^{v}(x) \in[0,1]$ defined in (6) is a factor that scales the outgoing flow of each input link at the junction $v$ such that the incoming flow to each output link is less than its supply. Thus, the model (4)-(8) maximizes the flow through links while ensuring that the outgoing flow at each link does not exceed the link's demand and the incoming flow does not exceed the link's supply. For entry link $\ell \in \mathcal{R}$, the incoming flow is additionally restricted to not exceed the exogenous demand $d_{\ell}$. The model further requires that, at each junction, the collection of outgoing flows for the input links is proportional to the collection of flow demand from the input links. This condition is referred to as proportional-priority, [21, 22], and differs from the constant priority model employed in $[7,8]$. From (4)-(8), we have forward invariance of the domain

$$
\mathcal{X} \triangleq \prod_{\ell \in \mathcal{L}}\left[0, \bar{x}_{\ell}\right]
$$

The model (4)-(8) is modified from the model proposed in [6] so that entry links have finite capacity. This is reasonable for traffic networks where entry links correspond to onramps with finite storage capacity.

Define the routing matrices $R_{\mathcal{O}} \in \mathbb{R}^{\mathcal{O} \times \mathcal{O}}$ and $R_{\mathcal{R}} \in$ $\mathbb{R}^{\mathcal{O} \times \mathcal{R}}$ elementwise as follows:

$$
\begin{aligned}
& {\left[R_{\mathcal{O}}\right]_{k \ell}=\left\{\begin{array}{ll}
\beta_{\rightarrow k} & \text { if } k \in \mathcal{L}_{\sigma(\ell)}^{\text {out }} \\
0 & \text { otherwise }
\end{array} \quad \forall \ell, k \in \mathcal{O}\right.} \\
& {\left[R_{\mathcal{R}}\right]_{k \ell}=\left\{\begin{array}{ll}
\beta_{\rightarrow k} & \text { if } k \in \mathcal{L}_{\sigma(\ell)}^{\text {out }} \\
0 & \text { otherwise }
\end{array} \quad \forall k \in \mathcal{O}, \forall \ell \in \mathcal{R} .\right.}
\end{aligned}
$$

Assumption 2 The matrix $\left(I-R_{\mathcal{O}}\right)$ is invertible.

Assumption 2 is equivalent to the assertion that eventually all vehicles will leave the network and is thus a natural assumption on the split ratios [27]. Let

$$
P=\left(I-R_{\mathcal{O}}\right)^{-1} R_{\mathcal{R}},
$$

that is, $P$ describes how the flow from entry links is routed through the network. As $\left(I-R_{\mathcal{O}}\right)^{-1}=I+R_{\mathcal{O}}+$ $R_{\mathcal{O}}^{2}+\ldots$, we have $P \geq 0$. Let

$$
f_{\ell}^{\mathrm{e}}= \begin{cases}d_{\ell} & \text { if } \ell \in \mathcal{R} \\ {[P d]_{\ell}} & \text { if } \ell \in \mathcal{O} .\end{cases}
$$

where $[P d]_{\ell}$ is the $\ell$ th entry of $P d$.

Assumption 3 The input flow $d=\left\{d_{\ell}\right\}_{\ell \in \mathcal{R}}$ satisfies

$$
f_{\ell}^{\mathrm{e}}<\Phi_{\ell}^{\text {crit }} \quad \forall \ell \in \mathcal{L} \text {. }
$$

Assumption 3 states that the network has adequate capacity to accomodate the input flow $d$, that is, $d$ is strictly feasible [14]. It follows from Assumption 3 that

$$
x_{\ell}^{\mathrm{e}} \triangleq\left(\Phi_{\ell}^{\text {out }}\right)^{-1}\left(f_{\ell}^{\mathrm{e}}\right)<x_{\ell}^{\text {crit }}
$$

for all $\ell \in \mathcal{L}$ constitutes an equilibrium of the traffic network dynamics (4)-(8). Indeed, for this case, $\alpha^{v}\left(x^{\mathrm{e}}\right)=1$ for all $v$ where we define $x^{\mathrm{e}}=\left\{x_{\ell}^{\mathrm{e}}\right\}_{\ell \in \mathcal{L}}$, that is, the outgoing flow on every link is equal to demand. A key result of this paper is that this equilibrium is unique and globally asymptotically stable for a class of networks defined subsequently.

\section{Nonmonotone behavior of traffic networks}

\subsection{Lack of monotonicity}

Consider the system $\dot{x}=G(x), x \in X \subseteq \mathbb{R}^{n}$ where $X$ is forward invariant and has convex interior. Suppose $G(\cdot)$ is locally Lipschitz and satisfies

$$
\frac{\partial G_{i}}{\partial x_{j}}(x) \geq 0 \quad \forall x \in X, \forall i \neq j
$$

whenever the derivative exists. Then the system $\dot{x}=$ $G(x)$ is order-preserving with respect to the positive orthant $\mathbb{R}_{\geq 0}^{n}$, that is,

$$
x(0) \leq y(0) \text { implies } x(t) \leq y(t) \quad \forall t \geq 0
$$

where $x(t), y(t)$ are solutions of the system with initial conditions $x(0), y(0)$. A dynamical system $\dot{x}=G(x)$ satisfying (16) is said to be monotone with respect to the positive orthant, or simply monotone [17, 26].

Traffic flow networks with no diverging junctions are monotone, as has been noted and studied in $[6,14]$. However, networks with diverging junctions are not monotone. To see this, consider a diverging junction $v$ and assume some link $\ell \in \mathcal{L}_{v}^{\text {out }}$ is the unique minimizer in (6) 
for some $x$ so that, for all $y$ in a neighborhood of $x$,

$$
\alpha^{v}(y)=\Phi_{\ell}^{\text {in }}\left(y_{\ell}\right)\left(\beta_{\rightarrow \ell} \sum_{k \in \mathcal{L}_{v}^{\text {in }}} \Phi_{k}^{\text {out }}\left(y_{k}\right)\right)^{-1} .
$$

It follows that, for all $k \in \mathcal{L}_{v}^{\text {out }}, k \neq \ell$ with $\sigma(k) \neq \sigma(\ell)$,

$$
\frac{\partial F_{k}}{\partial x_{\ell}}(x)=\frac{\partial f_{k}^{\text {in }}}{\partial x_{\ell}}(x)=\frac{\partial}{\partial x_{\ell}}\left(\frac{\beta_{\rightarrow k}}{\beta_{\rightarrow \ell}} \Phi_{\ell}^{\text {in }}\left(x_{\ell}\right)\right)<0,
$$

and thus the system is not monotone. We interpret (19) as follows: We assume the supply of downstream link $\ell$ is less than upstream demand due to congestion, and thus link $\ell$ inhibits flow through the junction. Therefore, an increase in the number of vehicles on link $\ell$ would worsen the congestion (decrease supply), and vehicles destined for link $\ell$ would further block flow to other outgoing links, causing a reduction in the incoming flow to these links. That is, the derivative of incoming flow to a downstream link $k \neq \ell$ with respect to link $\ell$ is nonzero and, in particular, is negative since $\Phi_{\ell}^{\text {in }}\left(x_{\ell}\right)$ is a decreasing function. Thus, lack of monotonicity is indeed expected for traffic networks and is relevant for transportation engineering because it is a primary explanation for why traffic control methods such as ramp metering are able to increase throughput.

Remark 1 It is standard to generalize the condition (16) to partial orders with respect to arbitrary orthants [16], and one may wonder if the traffic dynamics are monotone with respect to some alternative orthant order. The answer is negative; indeed, the relationship (19) holds for any pair of output links, and for a junction with at least three output links, this implies that the system is not monotone with respect to any orthant via the graphical condition in, e.g., [3, Proposition 2].

The phenomenon of downstream traffic blocking flow to other downstream links at a diverging junction is referred to as the first-in-first-out (FIFO) property, [10, 21 , and it is a feature of traffic flow that has been observed even on wide freeways with many lanes, [4, 24]. Some of the recent literature in dynamical flow models propose alternative modeling choices for diverging junctions, e.g., [5, 23], which ensures that the resulting dynamics are monotone and therefore do not exhibit this FIFO property.

\subsection{A weaker property: Mixed monotonicity}

The main result of this paper is that, while vehicular traffic networks are not monotone, they possess a weaker mixed monotonicity property. This property allows the traffic network dynamics to be embedded within a larger monotone system amenable to techniques for stability analysis of such systems.

We begin with a general characterization of mixed monotone systems which is a continuous-time analogue of the characterization in [25] and is closely related to recent results for nonmonotone interconnections of monotone systems, e.g., [1], as we discuss in Section 5.

Definition 1 (Mixed Monotone) The system $\dot{x}=$ $G(x), x \in X \subseteq \mathbb{R}^{n}$ where $X$ has convex interior and $G$ is locally Lipschitz is mixed monotone if there exists a locally Lipshitz continuous function $g(x, y)$ satisfying:

(1) $g(x, x)=G(x)$ for all $x \in X$

(2) $\frac{\partial g_{i}}{\partial x_{j}}(x, y) \geq 0$ for all $x, y \in X$ and all $i \neq j$ whenever the derivative exists

(3) $\frac{\partial g_{i}}{\partial y_{j}}(x, y) \leq 0$ for all $x, y \in X$ and all $i, j$ whenever the derivative exists.

The function $g(x, y)$ is called a decomposition function for the system.

For a mixed monotone system with decomposition function $g(x, y)$, it follows that the symmetric system

$$
\begin{aligned}
& \dot{x}=g(x, y) \\
& \dot{y}=g(y, x)
\end{aligned}
$$

is order-preserving with respect to the orthant $\mathbb{R}_{\geq 0}^{n} \times$ $\mathbb{R}_{\leq 0}^{n}$. We call (20)-(21) the embedding system for the mixed monotone system $\dot{x}=G(x)$. The embedding system plays a key role in the analysis to follow.

For all $\ell, k \in \mathcal{L}$, define

$$
s_{\ell k}= \begin{cases}1 & \text { if } \tau(k)=\tau(\ell) \text { and } k \neq \ell \\ 0 & \text { else }\end{cases}
$$

and for each $\ell \in \mathcal{L}$ and $x, y \in \mathbb{R}^{\mathcal{L}}$, let

$$
\begin{aligned}
\xi_{k}^{\ell}\left(x_{k}, y_{k}\right) & =s_{\ell k} y_{k}+\left(1-s_{\ell k}\right) x_{k} \quad \forall k \in \mathcal{L} \\
\xi^{\ell}(x, y) & =\left\{\xi_{k}^{\ell}\left(x_{k}, y_{k}\right)\right\}_{k \in \mathcal{L}} .
\end{aligned}
$$

Theorem 4 The traffic network model (4)-(8) is mixed monotone with decomposition function

$$
g_{\ell}(x, y)=f_{\ell}^{\text {in }}\left(\xi^{\ell}(x, y)\right)-f_{\ell}^{\text {out }}(x)
$$

Proof. We first note that $F_{\ell}(x)$ is Lipschitz continuous for each $\ell \in \mathcal{L}$; in the following, statements involving derivatives are interpreted to hold whenever the derivative exists.

For ease of notation, we interpret $f_{\ell}^{\text {in }}(x, y)=f_{\ell}^{\text {in }}\left(\xi^{\ell}(x, y)\right)$. It holds trivially that $g_{\ell}(x, x)=F_{\ell}(x)$ for all $\ell \in \mathcal{L}$. We 
now show that

$$
\begin{array}{ll}
\frac{\partial f_{\ell}^{\text {out }}}{\partial x_{k}}(x) \leq 0 & \forall x \in \mathcal{X}, \forall \ell \neq k \\
\frac{\partial f_{\ell}^{\text {in }}}{\partial x_{k}}(x, y) \geq 0 & \forall x, y \in \mathcal{X}, \forall \ell \neq k \\
\frac{\partial f_{\ell}^{\text {in }}}{\partial y_{k}}(x, y) \leq 0 & \forall x, y \in \mathcal{X}, \forall k
\end{array}
$$

which implies that $g_{\ell}(x, y)$ is indeed a valid decomposition function for the system and the system is mixed monotone, completing the proof.

To this end, first consider $k \in \mathcal{L}_{\sigma(\ell)}^{\text {out }}$. We have

$$
\begin{aligned}
& \frac{\partial f_{\ell}^{\text {out }}}{\partial x_{k}}(x)=\frac{\partial \alpha^{\sigma(\ell)}}{\partial x_{k}}(x) \Phi_{\ell}^{\text {out }}\left(x_{\ell}\right) \\
& \quad \in\left\{0, \frac{\Phi_{\ell}^{\text {out }}\left(x_{\ell}\right)}{\sum_{j \in \mathcal{L}_{\sigma(\ell)}^{\text {in }}} \beta_{\rightarrow k} \Phi_{j}^{\text {out }}\left(x_{j}\right)} \frac{d \Phi_{k}^{\text {in }}}{d x_{k}}\left(x_{k}\right)\right\} \leq 0 .
\end{aligned}
$$

If $k \in \mathcal{L}_{\sigma(\ell)}^{\text {in }}$, then (29) still holds and whenever $\partial \alpha^{\sigma(\ell)} / \partial x_{k} \neq 0$, there exists $m \in \mathcal{L}_{\sigma(\ell)}^{\text {out }}$ such that

$$
\begin{gathered}
\alpha^{\sigma(\ell)}(x)=\left(\sum_{j \in \mathcal{L}_{\sigma(\ell)}^{\text {in }}} \beta_{\rightarrow m} \Phi_{j}^{\text {out }}\left(x_{j}\right)\right)^{-1} \Phi_{m}^{\text {in }}\left(x_{m}\right) \\
\frac{\partial \alpha^{\sigma(\ell)}}{\partial x_{k}}(x)=-\frac{\frac{d \Phi_{k}^{\text {out }}}{d x_{k}}\left(x_{k}\right) \Phi_{m}^{\text {in }}\left(x_{m}\right)}{\beta_{\rightarrow m}\left(\sum_{j \in \mathcal{L}_{\sigma(\ell)}^{\text {in }}} \Phi_{j}^{\text {out }}\left(x_{j}\right)\right)^{2}} \leq 0,
\end{gathered}
$$

and thus (26) holds. Next, we have

$$
\frac{\partial f_{\ell}^{\text {in }}}{\partial x_{k}}(x, y)=\left\{\begin{array}{cc}
\beta_{\rightarrow \ell} \sum_{j \in \mathcal{L}_{\tau(\ell)}^{\text {in }}} \frac{\partial f_{j}^{\text {out }}}{\partial x_{k}}\left(\xi^{\ell}(x, y)\right) \\
\text { if } k \in \mathcal{L}_{\tau(\ell)}^{\text {in }}
\end{array}\right.
$$

by $(22)-(24)$. For $k \in \mathcal{L}_{\tau(\ell)}^{\text {in }}$, we have

$$
\begin{aligned}
\sum_{j \in \mathcal{L}_{\tau(\ell)}^{\text {in }}} \frac{\partial f_{j}^{\text {out }}}{\partial x_{k}}\left(\xi^{\ell}(x, y)\right) & =\frac{\partial \alpha^{\tau(\ell)}}{\partial x_{k}}\left(\xi^{\ell}(x, y)\right) \sum_{j \in \mathcal{L}_{\tau(\ell)}^{\text {in }}} \Phi_{j}^{\text {out }}\left(x_{j}\right) \\
& +\alpha^{\tau(\ell)}\left(\xi^{\ell}(x, y)\right) \frac{d \Phi_{k}^{\text {out }}}{d x_{k}}\left(x_{k}\right)
\end{aligned}
$$

If $\alpha^{\tau(\ell)} \neq 1$ on some neighborhood of $\xi^{\ell}(x, y)$, then there exists $m \in \mathcal{L}_{\tau(\ell)}^{\text {out }}$ such that

$$
\begin{aligned}
& \alpha^{\tau(\ell)}\left(\xi^{\ell}(x, y)\right)= \\
& \left(\sum_{j \in \mathcal{L}_{\tau(\ell)}^{\text {in }}} \beta_{\rightarrow m} \Phi_{j}^{\text {out }}\left(x_{j}\right)\right)^{-1} \Phi_{m}^{\text {in }}\left(\xi_{m}^{\ell}(x, y)\right) \\
& \frac{\partial \alpha^{\tau(\ell)}}{\partial x_{k}}\left(\xi^{\ell}(x, y)\right)=-\frac{\frac{d \Phi_{k}^{\text {out }}}{d x_{k}}\left(x_{k}\right) \Phi_{m}^{\text {in }}\left(\xi_{m}^{\ell}(x, y)\right)}{\beta_{\rightarrow m}\left(\sum_{j \in \mathcal{L}_{\tau(\ell)}^{\text {in }}} \Phi_{j}^{\text {out }}\left(x_{j}\right)\right)^{2}} .
\end{aligned}
$$

Then (34) evaluates to zero for this case. Therefore,

$$
\frac{\partial f_{\ell}^{\text {in }}}{\partial x_{k}}\left(\xi^{\ell}(x, y)\right) \in\left\{0, \beta_{\rightarrow \ell} \frac{d \Phi_{k}^{\text {out }}}{d x_{k}}\left(x_{k}\right)\right\} \geq 0,
$$

and thus (27) holds. Finally, we have

$$
\frac{\partial f_{\ell}^{\text {in }}}{\partial y_{k}}(x, y)= \begin{cases}\beta_{\rightarrow \ell} \frac{\partial \alpha^{\tau(\ell)}}{\partial x_{k}}\left(\xi^{\ell}(x, y)\right) \sum_{j \in \mathcal{L}_{\tau(\ell)}^{\text {in }}} \Phi_{j}^{\text {out }}\left(x_{j}\right) \\ \text { if } \tau(k)=\tau(\ell) \text { and } k \neq \ell \\ \text { else. }\end{cases}
$$

If $\frac{\partial \alpha^{\tau(\ell)}}{\partial x_{k}}\left(\xi^{\ell}(x, y)\right) \neq 0$ for some $k \neq \ell$ with $\tau(k)=\tau(\ell)$ then it must be that

$$
\frac{\partial \alpha^{\tau(\ell)}}{\partial x_{k}}\left(\xi^{\ell}(x, y)\right)=\left(\sum_{j \in \mathcal{L}_{\tau(\ell)}^{\text {in }}} \beta_{\rightarrow k} \Phi_{j}^{\text {out }}\left(x_{j}\right)\right)^{-1} \frac{d \Phi_{k}^{\text {in }}}{d x_{k}}\left(y_{k}\right) .
$$

We conclude that (28) holds because

$$
\frac{\partial f_{\ell}^{\text {in }}}{\partial y_{k}}(x, y) \in\left\{0, \frac{\beta_{\rightarrow \ell}}{\beta_{\rightarrow k}} \frac{d \Phi_{k}^{\text {in }}}{d x_{k}}\left(y_{k}\right)\right\} \leq 0
$$

\section{Asymptotic Behavior}

\subsection{Main result}

We now use Theorem 4 and the order-preserving properties of (20)-(21) to prove global stability of a particular class of traffic networks.

Definition 2 The connected graph $\mathcal{G}$ is said to be a polytree graph if the underlying undirected graph is acyclic.

The "underlying undirected graph" is the undirected graph that results from replacing each directed edge with an undirected edge between the same two nodes.

The class of networks that constitute polytree graphs is somewhat restrictive, as it does not allow cycles or multiple paths between two locations. However, polytrees still encompass a large class of relevant networks, such as a stretch of freeway with onramps and offramps, or a portion of a freeway network leading into (resp. out from) a large metropolitan area, which is useful for modeling the morning (resp. evening) commute patterns in the area. Furthermore, we show via a simple example in Section 4.2 that assuming the network is a polytree is necessary for the results presented here; this is an important observation in its own right as it demonstrates the limitations of the proposed approach and motivates additional techniques to overcome this limitation as discussed in Section 5. 
Theorem 5 The equilibrium $x^{\mathrm{e}}$ identified in (15) is globally asymptotically stable for polytree networks.

The proof proceeds as follows: We consider the embedding system (20)-(21) for the traffic flow dynamics and show that the extremal trajectory initialized at $\left(x_{0}, y_{0}\right)=(0, \bar{x})$ is such that $x(t)$ (resp. $\left.y(t)\right)$ is an increasing (resp. decreasing) function of time, converging to some limit $x^{*}$ ( resp. $\left.y^{*}\right)$. By monotonicity of the embedding system, we have that the omega limit set of the traffic flow dynamics lies in the interval defined by $x^{*}$ and $y^{*}$. By appealing to the specific form of the traffic flow dynamics (4)-(8), we then show that $x^{*}=y^{*}$.

Proof. We have

$$
\begin{aligned}
& 0 \leq g_{\ell}(0, \bar{x})= \begin{cases}d_{\ell} & \text { if } \ell \in \mathcal{R} \\
0 & \text { if } \ell \in \mathcal{O}\end{cases} \\
& 0 \geq g_{\ell}(\bar{x}, 0)= \begin{cases}0 & \text { if } \ell \in \mathcal{L} \backslash \mathcal{L}^{\text {sink }} \\
-\Phi_{\ell}^{\text {out }}\left(\bar{x}_{\ell}\right) & \text { if } \ell \in \mathcal{L}^{\text {sink }}\end{cases}
\end{aligned}
$$

Let $\leq_{C}$ be the order relation with respect to $C \triangleq \mathbb{R}_{\geq 0}^{n} \times$ $\mathbb{R}_{\leq 0}^{n}$, that is, $(x, y) \leq_{C}(\tilde{x}, \tilde{y})$ if and only if $x \leq \tilde{x}$ and $\tilde{y} \leq y$. Trivially, $(0, \bar{x}) \leq_{C}(\bar{x}, 0)$ and $(41)-(42)$ implies

$$
(g(\bar{x}, 0), g(0, \bar{x})) \leq_{C}(0,0) \leq_{C}(g(0, \bar{x}), g(\bar{x}, 0)) .
$$

Now consider the solution $(x(t), y(t))$ to $(20)-(21)$ with initial condition $(0, \bar{x})$. Since $(20)-(21)$ is orderpreserving with respect to $C$, the second inequality of (43) implies that $(x(t), y(t))$ is monotonically increasing with respect to $\leq_{C}[26$, Ch. 3, Prop. 2.1]. Symmetrically, $(y(t), x(t))$ is the solution to $(20)-(21)$ with initial condition $(\bar{x}, 0)$ and is monotonically decreasing by the first inequality of (43). It follows that $(x(t), y(t)) \rightarrow\left(x^{*}, y^{*}\right)$ for some $\left(x^{*}, y^{*}\right)$ an equilibrium of (20)-(21) satisfying

$$
x^{*} \leq x^{\mathrm{e}} \leq y^{*} .
$$

By symmetry, $\left(y^{*}, x^{*}\right)$ is also an equilibrium of (20)(21). That is, $f_{\ell}^{\text {in }}\left(\xi^{\ell}\left(x^{*}, y^{*}\right)\right)=f_{\ell}^{\text {out }}\left(y^{*}\right)$ for all $\ell \in \mathcal{L}$, and $f_{\ell}^{\text {in }}\left(\xi^{\ell}\left(y^{*}, x^{*}\right)\right)=f_{\ell}^{\text {out }}\left(x^{*}\right)$ for all $\ell \in \mathcal{L}$.

Consider a trajectory $z(t)$ of (4)-(8) with initial condition $z^{0} \in \mathcal{X}$ and let $\omega\left(z^{0}\right)$ denote the corresponding omega limit set. This induces the corresponding trajectory $(z(t), z(t))$ of the symmetric system $(20)-(21)$ with initial condition $\left(z^{0}, z^{0}\right)$. Since $(0, \bar{x}) \leq_{C}\left(z^{0}, z^{0}\right) \leq_{C}(\bar{x}, 0)$, it follows that $(x(t), y(t)) \leq_{C}(z(t), z(t)) \leq_{C}(y(t), x(t))$ for all $t \geq 0$, that is,

$$
x(t) \leq z(t) \leq y(t) \quad \forall t \geq 0 .
$$

We thus have $x^{*} \leq w \leq y^{*}$ for all $w \in \omega\left(z^{0}\right)$. We now show that $x^{*}=y^{*}=x^{\mathrm{e}}$.

Suppose $y^{*} \neq x^{\mathrm{e}}$. Recalling that $y^{*} \geq x^{\mathrm{e}}$, this implies there exists $\ell \in \mathcal{L}$ such that $y_{\ell}^{*}>x_{\ell}^{\mathrm{e}}$. By acyclicity of polytree networks, we assume, without loss of generality, that there does not exists $k \in \mathcal{L}_{\sigma(\ell)}^{\text {out }}$ such that $y_{k}^{*}>$ $x_{k}^{\mathrm{e}}$ (otherwise we could choose this downstream link $k$ instead of $\ell$ ). This implies that $\Phi_{k}^{\text {in }}\left(y_{k}^{*}\right)=\Phi_{k}^{\text {in }}\left(x_{k}^{\mathrm{e}}\right)$ for all $k \in \mathcal{L}_{\sigma(\ell)}^{\text {out }}$. Without loss of generality, we further assume that $f_{\ell}^{\text {out }}\left(y^{*}\right)>f_{\ell}^{\text {e }}$. Indeed, if this were not the case, then there must exist some downstream link with inadequate supply since $\Phi_{\ell}^{\text {out }}\left(y_{\ell}^{*}\right)>\Phi_{\ell}^{\text {out }}\left(x_{\ell}^{\mathrm{e}}\right)=f_{\ell}^{\mathrm{e}}$. That is, there exists $k \in \mathcal{L}_{\sigma(\ell)}^{\text {out }}$, for which $y_{k}^{*}=x_{k}^{\mathrm{e}}$, such that

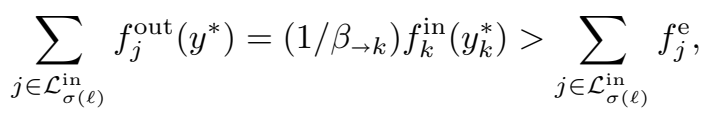

for which there must exist some $j \in \mathcal{L}_{\sigma(\ell)}^{\text {in }}, j \neq \ell$ with $f_{j}^{\text {out }}\left(y^{*}\right)>f_{j}^{\mathrm{e}}$, and we could choose $j$ instead of $\ell$.

We thus have $f_{\ell}^{\text {in }}\left(\xi^{\ell}\left(y^{*}, x^{*}\right)\right)=f_{\ell}^{\text {out }}\left(y^{*}\right)>f_{\ell}^{\text {e }}$. Define $\ell_{0} \triangleq \ell$ and, starting from $\ell_{0}$, choose inductively $\ell_{1}, \ell_{2}, \ldots, \ell_{n}$ to satisfy $\ell_{i} \in \mathcal{L}_{\tau\left(\ell_{i-1}\right)}^{\text {in }}$ for all $i$ (that is, link $\ell_{i}$ is upstream of link $\left.\ell_{i-1}\right)$ such that $f_{\ell_{i}}^{\text {out }}\left(y^{*}\right)>f_{\ell_{i}}^{\text {e }}$, until no additional upstream link satisfying this condition exists. Note it is possible that $\ell_{n}=\ell_{0}=\ell$. Thus

$$
f_{\ell_{n}}^{\text {in }}\left(\xi^{\ell_{n}}\left(y^{*}, x^{*}\right)\right)=f_{\ell_{n}}^{\text {out }}\left(y^{*}\right)>f_{\ell_{n}}^{\text {e }}
$$

and $f_{j}^{\text {out }}\left(y^{*}\right) \leq f_{j}^{\text {ee }}$ for all $j \in \mathcal{L}_{\tau\left(\ell_{n}\right)}^{\text {in }}$, which implies $\beta_{\rightarrow \ell_{n}} \sum_{j \in \mathcal{L}_{\tau(\ell)}^{\text {in }}} f_{j}^{\text {out }}\left(y^{*}\right)=f_{\ell_{n}}^{\text {in }}\left(y^{*}\right) \leq f_{\ell_{n}}^{\text {e }}$. With (47), this implies that $f_{\ell_{n}}^{\text {in }}\left(\xi^{\ell_{n}}\left(y^{*}, x^{*}\right)\right)>f_{\ell_{n}}^{\text {in }}\left(y^{*}\right)$. Thus there must exist $k$ with $\tau(k)=\tau\left(\ell_{n}\right)$ and $k \neq \ell_{n}$ such that the supply of link $k$ at state $y^{*}$ limits the outflow of the upstream links, that is, link $k$ is such that $\beta_{\rightarrow k} \sum_{j \in \mathcal{L}_{\tau(k)}^{\text {in }}} f_{j}^{\text {out }}\left(y^{*}\right)=$ $\Phi_{k}^{\text {in }}\left(y_{k}^{*}\right)$ for which $\Phi_{k}^{\text {in }}\left(y_{k}^{*}\right) \leq f_{k}^{\mathrm{e}}$.

Define $k_{0}=k$ and construct another sequence $k_{1}, k_{2}, \ldots, k_{n}$ satisfying $k_{i} \in \mathcal{L}_{\sigma\left(k_{i-1}\right)}^{\text {out }}$ for all $i$ (that is, link $k_{i}$ is downstream of link $k_{i-1}$ ) such that $\Phi_{k_{i}}^{\mathrm{in}}\left(y_{k_{i}}^{*}\right) \leq f_{k_{i}}^{\mathrm{e}}$, until no additional downstream link satisfying this condition exists. Note it is possible that $k_{n}=k_{0}=k$. It thus holds that $\Phi_{k_{n}}^{\text {in }}\left(y_{k_{n}}^{*}\right) \leq$ $f_{k_{n}}^{\mathrm{e}}$ and $\Phi_{j}^{\mathrm{in}}\left(y_{j}^{*}\right)>f_{j}^{\mathrm{e}}$ for all $j \in \mathcal{L}_{\sigma\left(k_{n}\right)}^{\text {out }}$. Recall $f_{k_{n}}^{\text {out }}\left(y^{*}\right) \leq \Phi_{k_{n}}^{\text {in }}\left(y_{k_{n}}^{*}\right)<\Phi_{k_{n}}^{\text {out }}\left(y_{k_{n}}^{*}\right)$, where the second inequality follows because $\Phi_{k_{n}}^{\text {in }}\left(y_{k_{n}}^{*}\right) \leq f_{k_{n}}^{\text {e }}$ and thus $y_{k_{n}}^{*}>x_{k_{n}}^{\text {crit }}$. Therefore there exists $j \in \mathcal{L}_{\sigma\left(k_{n}\right)}^{\text {out }}$ such that $\beta_{\rightarrow j} \sum_{m \in \mathcal{L}_{\sigma\left(k_{n}\right)}^{\text {in }}} f_{m}^{\text {out }}\left(y^{*}\right)=\Phi_{j}^{\text {in }}\left(y_{j}^{*}\right)$, but then there must exist $m \in \mathcal{L}_{\sigma\left(k_{n}\right)}^{\text {in }}$ such that $f_{m}^{\text {out }}\left(y^{*}\right)>f_{m}^{\text {e }}$ since $\Phi_{j}^{\text {in }}\left(y_{j}^{*}\right)>f_{j}^{\mathrm{e}}$, for which $y_{m}^{*}>x_{m}^{e}$. However, $m \neq \ell_{i}$ for any $\ell_{i} \in\left\{\ell_{0}, \ldots, \ell_{n}\right\}$ chosen previously, as this would 


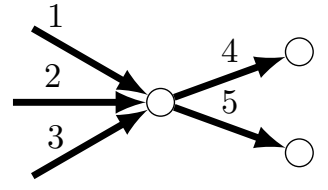

Fig. 2. An example consisting of a primary junction with three incoming links and two outgoing links.

imply the graph is not a polytree. Taking $\ell=m$, we could begin the process again, continuing indefinitely; since the graph is finite, we arrive at a contradiction, and thus we must have $y^{*}=x^{\mathrm{e}}$.

Now suppose $x^{*} \neq x^{\mathrm{e}}$, that is, there exists $\ell$ such that $x_{\ell}^{*}<x_{\ell}^{\mathrm{e}}$. Without loss of generality, assume there does not exist $k \in \mathcal{L}_{\tau(\ell)}^{\text {in }}$ such that $x_{k}^{*}<x_{k}^{\mathrm{e}}$. This implies

$$
f_{\ell}^{\text {out }}\left(x^{*}\right) \leq \Phi_{\ell}^{\text {out }}\left(x_{\ell}^{*}\right)<f_{\ell}^{\text {e }}
$$

Since $f_{\ell}^{\text {in }}\left(\xi^{\ell}\left(x^{*}, y^{*}\right)\right)=f_{\ell}^{\text {out }}\left(x^{*}\right)$ and

$$
\beta_{\rightarrow \ell} \sum_{k \in \mathcal{L}_{\tau(\ell)}^{\text {in }}} \Phi_{k}^{\text {out }}\left(x_{k}^{*}\right)=\beta_{\rightarrow \ell} \sum_{k \in \mathcal{L}_{\tau(\ell)}^{\text {in }}} \Phi_{k}^{\text {out }}\left(x_{k}^{\mathrm{e}}\right)=f_{\ell}^{\mathrm{e}}
$$

there must exists $j \in \mathcal{L}_{\tau(\ell)}^{\text {out }}, j \neq \ell$, such that $f_{\ell}^{\text {in }}\left(\xi^{\ell}\left(x^{*}, y^{*}\right)\right)=\left(\beta_{\rightarrow \ell} / \beta_{\rightarrow j}\right) \Phi_{j}^{\text {in }}\left(y_{j}^{*}\right)$, for which we must have $y_{j}^{*}>x_{j}^{\mathrm{e}}$, a contradiction since we have shown $y^{*}=x^{\mathrm{e}}$. As $x^{*}=y^{*}=x^{\mathrm{e}}$, we conclude that $\omega\left(z^{0}\right)=\left\{x^{\mathrm{e}}\right\}$ for all $z^{0} \in \mathcal{X}$, that is, the equilibrium $x^{\mathrm{e}}$ is globally attractive.

Finally, suppose the links are indexed $1, \ldots,|\mathcal{L}|$ such that the index of link $\ell$ is less than the index of each $k \in \mathcal{L}_{\sigma(\ell)}^{\text {out }}$ (such an indexing is always possible for polytree graphs). Then the Jacobian evaluated at the equilibrium, $(\partial F / \partial x)\left(x^{\mathrm{e}}\right)$, is lower triangular with respect to this indexing since $f_{\ell}^{\text {out }}\left(x^{\mathrm{e}}\right)=\Phi_{\ell}^{\text {out }}\left(x_{\ell}^{\mathrm{e}}\right)$ for all $\ell \in \mathcal{L}$. Additionally, the Jacobian contains strictly negative entries along the diagonal since $\Phi_{\ell}^{\text {out }}(\cdot)$ is strictly increasing, and it is therefore Hurwitz. Thus the equilibrium is locally asymptotically stable by, e.g., [19, Thereom 4.7] and therefore globally asymptotically stable since it is also globally attractive.

\subsection{Examples}

We first consider an example consisting of a single primary junction with multiple inputs and outputs which satisfies the polytree assumption.

Example 1 Let $\mathcal{R}=\{1,2,3\}$ and $\mathcal{O}=\{4,5\}$, and suppose the network consists of one primary junction as in Figure 2. We assume each link has supply and demand
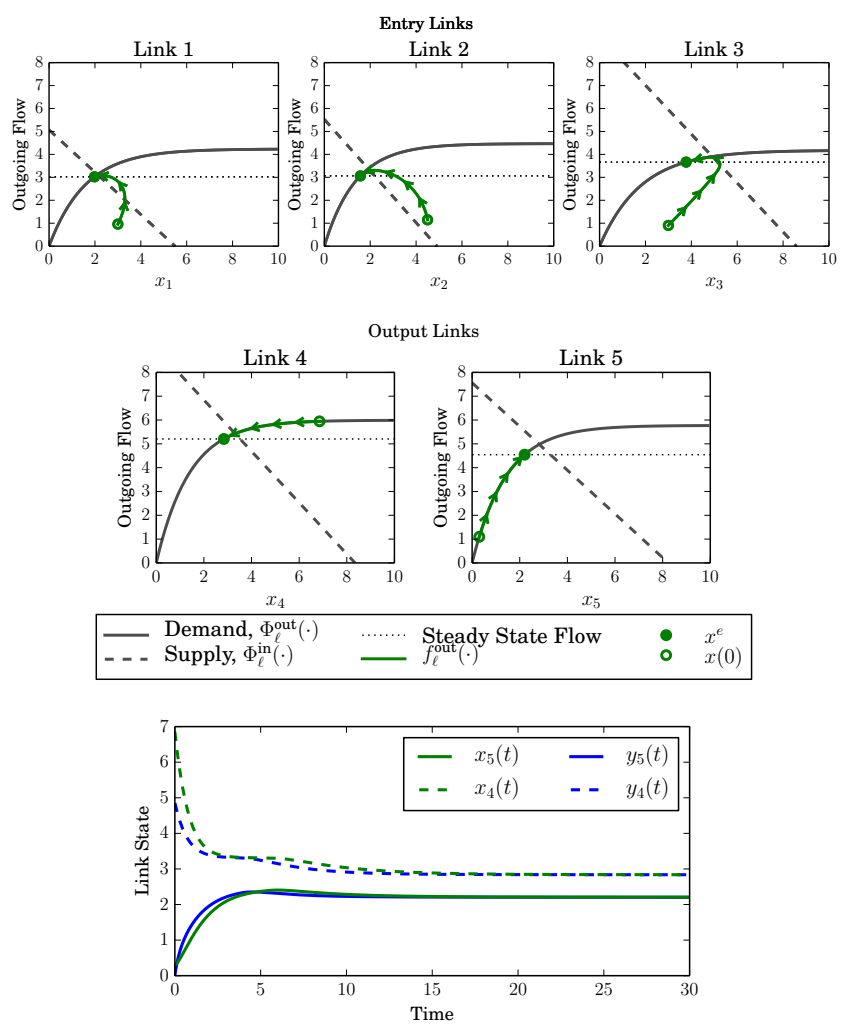

Fig. 3. Demand and supply functions for Example 1, along with an example trajectory of the system. The bottom plot shows the trace of the state of links 4 and 5 for the example trajectory (green), along with traces corresponding to a different initial condition (blue). These traces demonstrates that the system is not monotone since the solid lines cross.

functions of the form

$$
\begin{aligned}
\Phi_{\ell}^{\text {in }}\left(x_{\ell}\right) & =\zeta_{\ell}-m_{\ell} x_{\ell} \\
\Phi_{\ell}^{\text {out }}\left(x_{\ell}\right) & =\gamma_{\ell}\left(1-\exp \left(-\delta_{\ell} x_{\ell}\right)\right)
\end{aligned}
$$

where $\zeta_{\ell} \in[4,10], m_{\ell} \in[0.75,1.25], \gamma_{\ell} \in[4,6], \delta_{\ell} \in$ $[0.25,0.75]$, and each parameter is chosen uniformly randomly from the given set for the simulation. The split ratios $\beta_{\rightarrow 4}$ and $\beta_{\rightarrow 5}$ are also chosen randomly. The exogenous input satisfies $d_{\ell} \in[3,5]$ and is chosen uniformly randomly, and we ensure $\left\{d_{\ell}\right\}_{\ell \in \mathcal{R}}$ satisfies Assumption 3. Figure 3 shows plots of the demand and supply functions, along with a sample trajectory $x(t)$ of the system. We see that the system converges to the unique equilibrium, and furthermore $f_{\ell}^{\text {out }}(x(t))$ is strictly less than $\Phi_{\ell}^{\text {out }}\left(x_{\ell}(t)\right)$ for $\ell \in \mathcal{R}$ for most of the sample trajectory, indicating that the flow is constrained by a downstream link. In particular, link 4 restricts the flow of upstream links and results in nonmonotone behavior. We see this in the lower plot of the figure, which shows $x_{4}(t)$ and $x_{5}(t)$, along with $y_{4}(t)$ and $y_{5}(t)$ for another trajectory with initial condition satisfying $y_{0} \leq x_{0}$. Since the trace of $x_{5}(t)$ and $y_{5}(t)$ cross, the system is not monotone. 


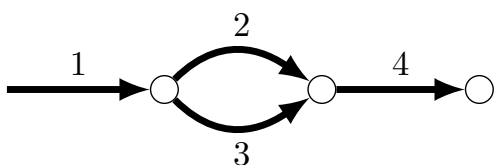

Fig. 4. An example that is not a polytree network for which Theorem 5 does not apply. In particular, the symmetric system (20)-(21) admits additional equilibria that do not correspond to an equilibrium of the traffic network, demonstrating the necessity of the polytree condition and illuminating an important limitation of the proposed technique.

We next present an example network that is not a polytree, for which $y^{*} \neq x^{\mathrm{e}}$ if we attempt to apply the proof technique of Theorem 5. This example shows that the polytree condition in Theorem 5 is tight and illuminates the limitations of applying monotonicity results to establish convergence in nonmonotone traffic flow networks. We discuss these limitations in further detail below.

Example 2 Consider the traffic network shown in Figure 4 and assume

$$
\begin{aligned}
\Phi_{\ell}^{\text {out }}\left(x_{\ell}\right) & =x_{\ell}, & & \ell \in\{1,2,3,4\} \\
\Phi_{\ell}^{\text {in }}\left(x_{\ell}\right) & =30-x_{\ell}, & & \ell \in\{1,2,4\} \\
\Phi_{3}^{\text {in }}\left(x_{3}\right) & =100-x_{3}, & &
\end{aligned}
$$

and $\beta_{\rightarrow 2}=\beta_{\rightarrow 3}=1 / 2, \beta_{\rightarrow 4}=1$, and $d_{1}=10$. Then

$$
x^{\mathrm{e}}=(10,5,5,10), \quad f^{\mathrm{e}}=(10,5,5,10)
$$

constitutes the unique equilibrium and equilibrium flow. However, taking $x^{*}=x^{\mathrm{e}}$ and $y^{*}=(20,25,50,15)$, we have that $g\left(x^{*}, y^{*}\right)=g\left(y^{*}, x^{*}\right)=0$ for $g(x, y)$ as given in Theorem 4 , that is, $\left(x^{*}, y^{*}\right)$ and $\left(y^{*}, x^{*}\right)$ constitute equilibria of the symmetric system. Nonetheless, simulations indicate that $x^{\mathrm{e}}$ is indeed globally asymptotically stable.

\section{Discussion and Conclusions}

We showed that traffic flow networks are not monotone due to multi-output junctions. Nevertheless, we observed in Theorem 4 that increasing the number of vehicles on one outgoing link can only decrease incoming flow to adjacent outgoing links, and thus the dynamics are mixed monotone and amenable to an embedding into a higher order, symmetric monotone system.

Theorem 5 used monotonicity of this embedding system and proved convergence to the equilibrium of the original nonmonotone system. In $[1,2,11,12]$, a similar technique is used to establish general small-gain theorems for nonmonotone interconnections of monotone subsystems. Other approaches to proving stability of flow networks rely on a one-norm contraction property that is partially due to conservation of mass in the network but requires monotone dynamics $[5,6]$. It is not clear how techniques that rely on contraction properties can be extended to mixed monotone systems, even after embedding such systems in a symmetric monotone system as in (20)-(21). However, this is a natural area to explore for overcoming the polytree condition in Theorem 5 .

\section{References}

[1] Angeli, D., Enciso, G. A., and Sontag, E. D. (2014). A small-gain result for orthant-monotone systems under mixed feedback. Systems \& Control Letters, 68:9-19.

[2] Angeli, D. and Sontag, E. (2003). Monotone control systems. IEEE Transactions on Automatic Control, 48(10):1684-1698.

[3] Angeli, D. and Sontag, E. (2004). Interconnections of monotone systems with steady-state characteristics. In Optimal control, stabilization and nonsmooth analysis, pages 135-154. Springer.

[4] Cassidy, M. J., Anani, S. B., and Haigwood, J. M. (2002). Study of freeway traffic near an off-ramp. Transportation Research Part A: Policy and Practice, 36(6):563-572.

[5] Como, G., Lovisari, E., and Savla, K. (2015). Throughput optimality and overload behavior of dynamical flow networks under monotone distributed routing. IEEE Transactions on Control of Network Systems, 2(1):57-67.

[6] Coogan, S. and Arcak, M. (2014). Dynamical properties of a compartmental model for traffic networks. In Proceedings of the 2014 American Control Conference, pages 2511-2516.

[7] Coogan, S. and Arcak, M. (2015). Efficient finite abstraction of mixed monotone systems. In Proceedings of the 18th International Conference on Hybrid Systems: Computation and Control, pages 58-67.

[8] Coogan, S., Aydin Gol, E., Arcak, M., and Belta, C. (2015). Controlling a network of signalized intersections from temporal logical specifications. In Proceedings of the 2015 American Control Conference, pages 3919-3924.

[9] Daganzo, C. F. (1994). The cell transmission model: A dynamic representation of highway traffic consistent with the hydrodynamic theory. Transportation Research Part B: Methodological, 28(4):269-287.

[10] Daganzo, C. F. (1995). The cell transmission model, part II: Network traffic. Transportation Research Part B: Methodological, 29(2):79-93.

[11] Enciso, G., Smith, H., and Sontag, E. (2006). Nonmonotone systems decomposable into monotone systems with negative feedback. Journal of Differential Equations, 224(1):205-227.

[12] Enciso, G. and Sontag, E. (2006). Global attractivity, I/O monotone small-gain theorems, and biological delay systems. Discrete and Continuous Dynamical Systems, 14(3):549.

[13] Gomes, G. and Horowitz, R. (2006). Optimal freeway ramp metering using the asymmetric cell trans- 
mission model. Transportation Research Part C: Emerging Technologies, 14(4):244-262.

[14] Gomes, G., Horowitz, R., Kurzhanskiy, A. A., Varaiya, P., and Kwon, J. (2008). Behavior of the cell transmission model and effectiveness of ramp metering. Transportation Research Part C: Emerging Technologies, 16(4):485-513.

[15] Gouzé, J. and Hadeler, K. (1994). Monotone flows and order intervals. Nonlinear World, 1:23-34.

[16] Hirsch, M. and Smith, H. (2005). Monotone dynamical systems. Handbook of differential equations: Ordinary differential equations, 2:239-357.

[17] Hirsch, M. W. (1985). Systems of differential equations that are competitive or cooperative II: Convergence almost everywhere. SIAM Journal on Mathematical Analysis, 16(3):423-439.

[18] Jacquez, J. A. and Simon, C. P. (1993). Qualitative theory of compartmental systems. SIAM Review, 35(1):43-79.

[19] Khalil, H. K. (2002). Nonlinear Systems. Prentice Hall, third edition.

[20] Kulenovic, M. and Merino, O. (2006). A global attractivity result for maps with invariant boxes. Discrete and Continuous Dynamical Systems Series B, 6(1):97.

[21] Kurzhanskiy, A. A. and Varaiya, P. (2010). Active traffic management on road networks: A macroscopic approach. Philosophical Transactions of the Royal Society A: Mathematical, Physical and Engineering Sciences, 368(1928):4607-4626.

[22] Kurzhanskiy, A. A. and Varaiya, P. (2012). Guaranteed prediction and estimation of the state of a road network. Transportation Research Part C: Emerging Technologies, 21(1):163-180.

[23] Lovisari, E., Como, G., and Savla, K. (2014). Stability of monotone dynamical flow networks. In Proceedings of the 53rd Conference on Decision and Control, pages 2384-2389.

[24] Munoz, J. C. and Daganzo, C. F. (2002). The bottleneck mechanism of a freeway diverge. Transportation Research Part A: Policy and Practice, 36(6):483505 .

[25] Smith, H. (2008). Global stability for mixed monotone systems. Journal of Difference Equations and Applications, 14(10-11):1159-1164.

[26] Smith, H. L. (1995). Monotone dynamical systems: An introduction to the theory of competitive and cooperative systems. American Mathematical Society.

[27] Varaiya, P. (2013). The max-pressure controller for arbitrary networks of signalized intersections. In Advances in Dynamic Network Modeling in Complex Transportation Systems, pages 27-66. Springer. 